\title{
Investigation of dynamic elastic deformation of parts processed by fused deposition modeling additive manufacturing
}

\author{
Mohamed, Omar A. ${ }^{\mathrm{a},{ }^{*}}$, Masood, Syed H. ${ }^{\mathrm{a}}$, Bhowmik, Jahar L. ${ }^{\mathrm{b}}$ \\ a Department of Mechanical and Product Design Engineering, Swinburne University of Technology, Hawthorn, Australia \\ bepartment of Statistics, Data Science and Epidemiology, Swinburne University of Technology, Hawthorn, Australia
}

\begin{abstract}
A B S T R A C T
Fused deposition modeling (FDM) has been recognized as an effective technology to manufacture 3D dimensional parts directly from a digital computer aided design (CAD) model in a layer-by-layer style. Although it has become a significantly important manufacturing process, but it is still not well accepted additive manufacturing technology for load-carrying parts under dynamic and cyclic conditions due to many processing parameters affecting the part properties. The purpose of this study is to characterize the FDM manufactured parts by detecting how the individual and interactive FDM process parameters will influence the performance of manufactured products under dynamic and cyclic conditions. Experiments were conducted through fractional factorial design and artificial neural network (ANN). Effect of each parameter on the dynamic modulus of elasticity was investigated using analysis of variance (ANOVA) technique. Furthermore, optimal processing parameters were determined and validated by conducting verification experiment. The results showed that both ANN and fractional factorial models provided good quality predictions, yet the ANN showed the superiority of a properly trained ANN in capturing the nonlinear relationship of the system over fractional factorial for both data fitting and estimation capabilities.
\end{abstract}

\section{ARTICLE INFO}

Keywords:

Additive manufacturing

Fused deposition modeling (FDM)

Dynamic modulus of elasticity

Fraction factorial design

Artificial neural network (ANN)

Process parameters

Analysis of variance (ANOVA)

${ }^{*}$ Corresponding author:

Omar.Ahmed.Mohamed@outlook.com (Mohamed, Omar A.)

Article history:

Received 18 June 2016

First Revision 22 August 2016

Second Revision 28 August 2016

Accepted 29 August 2016

\section{References}

[1] Mohamed, O.A., Masood, S.H., Bhowmik, J.L. (2015). Optimization of fused deposition modeling process parameters: A review of current research and future prospects. Advances in Manufacturing, Vol. 3, No. 1, 42-53, doi: $10.1007 / \mathrm{s} 40436-014-0097-7$.

[2] Wang, C.C., Lin, T.-W., Hu, S.-S. (2007). Optimizing the rapid prototyping process by integrating the Taguchi method with the Gray relational analysis, Rapid Prototyping Journal, Vol. 13, No. 5, 304-315, doi: $10.1108 / 13552540710824814$.

[3] Onwubolu, G.C., Rayegani, F. (2014). Characterization and Optimization of Mechanical Properties of ABS Parts Manufactured by the Fused Deposition Modelling Process, International Journal of Manufacturing Engineering, Volume 2014, Article ID 598531, 13 pages, doi: 10.1155/2014/598531.

[4] Sood, A.K., Ohdar, R., Mahapatra, S. (2010). Parametric appraisal of mechanical property of fused deposition modelling processed parts, Materials \& Design, Vol. 31, No. 1, 287-295, doi: 10.1016/j.matdes.2009.06.016.

[5] Jaya Christiyan, K.G., Chandrasekhar, U., Venkateswarlu, K. (2016). A study on the influence of process parameters on the Mechanical Properties of 3D printed ABS composite, IOP Conference Series: Materials Science and Engineering, Vol. 114, No. 1, 012109, doi: 10.1088/1757-899X/114/1/012109. 
[6] Impens, D., Urbanic, R.J. (2015). Assessing the impact of post-processing variables on tensile and compression characteristics for 3D printed components, IFAC-PapersOnLine, Vol. 48, No. 3, 652-657, doi: 10.1016/i.ifacol. 2015.06.156.

[7] Lanzotti, A., Grasso, M., Staiano, G., Martorelli, M. (2015). The impact of process parameters on mechanical properties of parts fabricated in PLA with an open-source 3-D printer, Rapid Prototyping Journal, Vol. 21, No. 5, 604-617, doi: 10.1108/RPJ-09-2014-0135.

[8] Arivazhagan, A., Masood, S., Sbarski, I. (2011). Dynamic mechanical analysis of FDM rapid prototyping processed polycarbonate material, In: Proceedings of the 69th Annual Technical Conference of the Society of Plastics Engineers 2011, Society of Plastics Engineers, Boston, Massachusetts, United States, USA, 950-955.

[9] Arivazhagan, A., Masood, S. (2012). Dynamic mechanical properties of ABS material processed by fused deposition modelling, International Journal of Engineering Research and Applications, Vol. 2, No. 3, 2009-2014.

[10] Eriksson, L., Johansson, E., Kettaneh-Wold, N., Wikström, C., Wold, S. (2008). Design of experiments: Principles and applications, $3^{\text {rd }}$ edition, MKS Umetrics AB, Umeå, Sweden.

[11] Douglas, C.M. (2012). Design and analysis of experiments, $8^{\text {th }}$ edition, John Wiley \& Sons, USA.

[12] ASTM D5418-07. (2007). Standard test method for plastics: Dynamic mechanical properties: In flexure (Dual Cantilever Beam), ASTM International, West Conshohocken.

[13] DMA2980 (2002). Dynamic mechanical analysis. Operator's manual, TA Instrument, New Castle, pp. 4-8.

[14] Menard, K.P. (2008). Dynamic mechanical analysis: A practical introduction, CRC press, $2^{\text {nd }}$ edition, Taylor \& Francis Group, Florida, USA, doi: 10.1201/9781420053135. 


\title{
Raziskava dinamične elastične deformacije delov, narejenih s pomočjo dodajalne izdelovalne tehnologije neprekinjenega ciljnega nalaganja
}

\author{
Mohamed, Omar A. ${ }^{a,}{ }^{,}$, Masood, Syed H. ${ }^{a}$, Bhowmik, Jahar L. ${ }^{b}$ \\ ${ }^{a}$ Department of Mechanical and Product Design Engineering, Swinburne University of Technology, Hawthorn, Australia \\ ${ }^{b}$ Department of Statistics, Data Science and Epidemiology, Swinburne University of Technology, Hawthorn, Australia
}

\begin{abstract}
POVZETEK
Neprekinjeno ciljno nalaganje (angl. FDM) se je izkazalo kot učinkovita tehnologija pri izdelavi 3D-kosov po načinu gradnje plast za plastjo, neposredno iz digitalnih CAD-modelov. Čeprav je FDM postal pomemben izdelovalni postopek, še vedno ni dovolj dobro sprejet ko gre za kose, ki so podvrženi povečani obremenitvi, še posebej pa dinamičnim in cikličnim obremenitvam, saj številni procesni parametri pri izdelavi kosov bistveno vplivajo na njihove lastnosti. Namen pričujoče raziskave je raziskati kako vpliva posamezni procesni parameter - prav tako pa soodvisnost več parametrov - na lastnosti kosov pod dinamičnimi in cikličnimi obremenitvami. Eksperiment smo izvedli s pomočjo delnega faktorskega načrta in umetnih nevronskih mrež (angl. ANN). Vpliv vsakega parametra na dinamični modul elastičnosti smo raziskali s pomočjo analize variance. Poleg tega smo določili optimalne procesne parametre in jih potrdili z izvedbo testov za verifikacijo. Rezultati so pokazali, da obe metodi, tako ANN kot tudi delni faktorski načrt, omogočata dobro kakovost napovedovanja, vendar pa smo ugotovili, da metoda ANN uspešneje opiše nelinearne relacije v preučevanem sistemu kot pa delni faktorski načrt.
\end{abstract}

\section{PODATKI O ČLANKU}

Ključne besede:

Dodajalni izdelovalni postopek

Neprekinjeno ciljno nalaganje (FDM)

Dinamični modul elastičnosti

Delni faktorski načrt

Umetne nevronske mreže (ANN)

Procesni parametri

Analiza variance (ANOVA)

*Kontaktna oseba:

Omar.Ahmed.Mohamed@outlook.com (Mohamed, Omar A.)

Zgodovina članka:

Prejet 18. junija 2016

Prvič popravljen 22. avgusta 2016

Drugič popravljen 28. avgusta 2016

Sprejet 29. avgusta 2016 